\title{
Arborescences
}

Revue d'études françaises

\section{Variation sociophonétique dialectale et stylistique : quelle est la langue cible en français langue seconde à Montréal ?}

\section{Monelle Guertin}

Numéro 7, décembre 2017

La norme orale en français laurentien

URI : https://id.erudit.org/iderudit/1050969ar

DOI : https://doi.org/10.7202/1050969ar

Aller au sommaire du numéro

Éditeur(s)

Département d'études françaises, Université de Toronto

ISSN

1925-5357 (numérique)

Découvrir la revue

Citer cet article

Guertin, M. (2017). Variation sociophonétique dialectale et stylistique : quelle est la langue cible en français langue seconde à Montréal ? Arborescences, (7), 67-89. https://doi.org/10.7202/1050969ar

\section{Résumé de l'article}

La variété de français parlée dans l'Hexagone a été par le passé considérée comme un modèle de «bon français ", tandis que la variété québécoise s'est trouvée dépeinte de façon moins prestigieuse. En enseignement des langues secondes, ces opinions ont un impact sur les choix et les perceptions des apprenants envers de la langue cible. Plusieurs chercheurs ont étudié ces perceptions dans une comparaison France-Québec. Cette étude propose de vérifier si la variation stylistique propre à chaque variété dialectale affecte les réactions envers le français de France et celui du Québec de la même manière que les variétés le font, pour des apprenants de français langue seconde à Montréal. Selon la technique du locuteur masqué, les participants ont écouté et réagi à des locutrices natives du français parisien et du français québécois produisant exactement le même contenu linguistique dans deux situations différentes, formelle et informelle. Les résultats montrent que la variation stylistique a eu une importance considérable dans les perceptions et les choix des participants. Parallèlement, les données révèlent un amalgame réalisé entre, d'une part, langue formelle et français européen et, d'autre part, langue informelle et français québécois.
Tous droits réservés @ Département d'études françaises, Université de Toronto, 2018
Ce document est protégé par la loi sur le droit d'auteur. L'utilisation des services d'Érudit (y compris la reproduction) est assujettie à sa politique d'utilisation que vous pouvez consulter en ligne. 


\section{SOMMAIRE}

1 Marie-Hélène Côté, Université de Lausanne

Anne-José Villeneuve, Université de l'Alberta Introduction

17 Davy Bigot, Université Concordia

Regard rétrospectif sur la norme du français québécois oral

33 Marie-Hélène Côté, Université de Lausanne

Wim Remysen, Université de Sherbrooke

Le "bon usage du français au Québec» selon le Multidictionnaire de la langue française: le cas de la prononciation

49 Anne-José Villeneuve, Université de l'Alberta

Normes objectives et variation socio-stylistique: le français québécois parlé en contexte d'entrevues télévisées

67 Monelle Guertin, Université du Québec à Montréal

Variation sociophonétique dialectale et stylistique: quelle est la langue cible en français langue seconde à Montréal?

90 Suzie Beaulieu, Université Laval

Leif French, Sam Houston State University

Samuel Gagnon, Université Laval

I'veulent-tu parler comme nous-autres? Opinions d'apprenants sur la forme interrogative totale en français laurentien 


\title{
Variation sociophonétique dialectale et stylistique: quelle est la langue cible en français langue seconde à Montréal ? ${ }^{1}$
}

\author{
Monelle Guertin, Université du Québec à Montréal
}

\section{Résumé}

La variété de français parlée en France a été par le passé considérée comme un modèle de «bon français", tandis que la variété québécoise s'est trouvée dépeinte de façon moins prestigieuse. En enseignement des langues secondes, ces opinions ont un impact sur les choix et les perceptions des apprenants envers de la langue cible. Plusieurs chercheurs ont étudié ces perceptions dans une comparaison France - Québec. Cette étude propose de vérifier si la variation stylistique propre à chaque variété dialectale affecte les réactions envers le français de France et celui du Québec de la même manière que les variétés le font, pour des apprenants de français langue seconde à Montréal. Selon la technique du locuteur masqué, les participants ont écouté et réagi à des locutrices natives du français parisien et du français montréalais produisant exactement le même contenu linguistique dans deux situations différentes, formelle et informelle. Les résultats montrent que la variation stylistique a eu une importance considérable dans les perceptions et les choix des participants. Parallèlement, les données révèlent un amalgame réalisé entre, d'une part, langue formelle et français européen et, d'autre part, langue informelle et français québécois.

\section{Introduction}

Au Québec, le «français de France» a par le passé été désigné comme le «bon» modèle linguistique, celui auquel se référer; la variété québécoise, en revanche, a régulièrement hérité de jugements dépréciatifs (Lambert et al. 1960; Lambert 1967; d'Anglejan et Tucker 1973; Lappin 1982; Bouchard 2002). Aujourd'hui, le débat n'est pas tout à fait clos à propos de la nature, endogène ou exogène, que devrait revêtir la norme linguistique québécoise (Maurais 2008; Bigot 2011; Brousseau 2011; Bigot et Papen 2013). Cette hésitation connait des échos en classe de français langue seconde (FLS), où le français québécois serait souvent dévalué (Archambault et Corbeil 1982; Calinon 2009).

La présente étude a pour objets les perceptions et les préférences d'apprenants de FLS à Montréal à l'égard de la variation sociophonétique présente entre les variétés du français québécois (FQ) et du français de France $(\mathrm{FF})$, ainsi qu'envers la variation stylistique propre à chacune de ces variétés ${ }^{2}$. De ce fait, cette recherche pose la question, moins souvent abordée dans les travaux précédents, de l'importance de la variation stylistique (et non uniquement dialectale) pour les attitudes des apprenants de FLS. La notion de style à laquelle je me réfère dans cette étude est ancrée dans la proposition

1. Je tiens à remercier sincèrement Philip Comeau, Anne-José Villeneuve, Marie-Hélène Côté, Lucie Ménard, Carolanne Paquin-Drouin et les évaluateurs anonymes pour leurs commentaires sur les différentes versions de cet article. Je remercie aussi chaleureusement Daphnée Simard pour la direction de mon mémoire et Lucie Ménard, à nouveau, pour son aide concernant les mesures acoustiques. Merci au CRLEC et au CRLMB pour leur appui financier.

2. FF, pour «français de France», fait référence au français dit hexagonal, plus particulièrement au français parisien, tandis que FQ, pour «français québécois», fait référence, dans la présente étude, au français montréalais. 
labovienne, à savoir une variation linguistique chez un même locuteur, induite par l'attention portée à sa langue en fonction du degré de formalité de la situation (Labov 1972).

La variation sociolinguistique est réputée susciter chez l'auditeur des jugements sociaux, lesquels reflètent son attitude envers les langues, les variétés et les locuteurs (Giles et Powesland 1975). Pour Moyer (2013), l'«accent» d'un locuteur offre une prise instantanée au jugement de l'auditeur. Gardner (1985) définit l'attitude comme une réaction évaluative à un objet, produite à partir des croyances et des opinions de l'individu à propos de cet objet (Gardner 1985: 9). Pour Lasagabaster (2006: 395), en langue seconde (L2), les enseignants, les institutions du pays d'accueil et les opinions véhiculées sur la langue cible (LC) par la société et les médias sont aux premiers rangs des sources d'influence sur les attitudes d'apprenants de L2. Tremblay et Gardner (1995: 515) expliquent qu' une attitude oriente les buts de l'apprenant dans son apprentissage. La perception qu'un apprenant peut avoir d'une langue ou d'une variété influence sa motivation et, finalement, son succès (Gardner et al. 1997: 353).

Les travaux de Maurais (2008), Saint-Laurent (2008), Calinon (2009) et Kircher (2012) sur les opinions d'immigrants, d'allophones ${ }^{3}$ et d'apprenants de FLS au Québec montrent une tendance à la dépréciation du FQ et à la valorisation, voire à l'idéalisation, du FF. Dans la présente étude, les attitudes d'apprenants de FLS sont examinées à l'aide de la «matched-guise technique» ou technique du locuteur masqué (Lambert et al. 1960). À l'écoute de stimuli enregistrés avec des locutrices natives du FQ et du FF en situations formelle (FOR) et informelle (INF), les participants ont répondu à un questionnaire élicitant, d'une part, leur perception de la provenance des locutrices - examinée en fonction du style - et, d'autre part, leur préférence en matière de LC. L'objectif de l'étude est de vérifier si une variété ou un style peut représenter pour les participants la «bonne» LC. Les résultats montrent une préférence pour une langue FOR, tout en laissant paraître, parallèlement, l'existence d'un amalgame partiel entre FQ et langue non standard, d'un côté, et FF et langue standard, de l'autre.

Après une brève mise en contexte (sections 2 et 3), les résultats d'études antérieures et les questions de recherche seront présentés (section 4). La méthode utilisée sera ensuite expliquée (sections 5), avec une attention particulière portée à la description de la variation sociophonétique présente dans les stimuli (sections 5.2.3 et 5.2.4). Puis les résultats et une discussion en lien avec chaque question de recherche seront présentés (section 6), pour terminer avec des pistes futures et une conclusion (section 7).

\section{Immigration et FLS au Québec}

Entre 2009 et 2013, chaque année en moyenne 52442 personnes d'origines diverses se sont installées au Québec, dont 57,4\% avaient une "connaissance du français» (MIDI 2014: 7, 9). Statistique Canada (2011) chiffre à 612945 le nombre de personnes immigrantes pour l'agglomération de Montréal, soit environ $33 \%$ de sa population totale. Ces immigrants vivent les enjeux de l'intégration sociale et professionnelle et parmi eux, un certain nombre, pour y parvenir, entreprend l'apprentissage du français (CSLF 2007). Mais de quel «français»? Cette question a été abordée dans une perspective didactique, notamment par F. Mougeon (1995) et Detey et al. (2010), ce qui a permis d'insister sur la nécessité, pour les apprenants de FLS, d'être sensibilisés à la variation stylistique et dialectale. Or, une forme "d'idéologie du standard» (Gadet 2007) ou une tendance à croire que la langue correcte est issue d'une seule variété, en l'occurrence le FF, demeure présente dans le matériel didactique, les

3. Personnes dont la langue maternelle n'est ni le français ni l'anglais. 
programmes et les attitudes de certains enseignants, tandis que le FQ est qualifié de variété familière ou populaire (Archambault et Corbeil 1982; Lepicq et Bouhris 1995; Calinon 2009) ${ }^{4}$. Néanmoins, le Québec n'est pas en reste par rapport à une lacune dans l'enseignement de variantes non standards et de particularités locales. Valdman (2000) et Paternostro (2014) font ainsi remarquer, pour l'enseignement du français langue étrangère aux États-Unis et en Europe, un manque à combler concernant l'enseignement de la variation diatopique et diaphasique du français ${ }^{5}$.

\section{Une norme du français québécois?}

En 1990, le Conseil supérieur de la langue française (CSLF) pressait le Québec d'entreprendre une description en règle de ses usages linguistiques, constatant dans les ouvrages de référence disponibles, et principalement européens, «une grave lacune: les ouvrages de référence actuellement sur le marché ne répondent pas pleinement aux besoins des Québécois parce qu'ils ne s'appuient pas sur une description complète et scientifique de leurs usages" (CSLF 1990).

En 1995, Cajolet-Laganière et Martel (1995: 158) précisaient:

Nous sommes convaincus qu'un modèle linguistique français, mais français québécois, fait consensus au Québec. Nous pratiquons le français québécois standard tout en croyant suivre le modèle français. Or, ce modèle québécois n'est décrit nulle part, à l'heure actuelle.

Bigot (2011: 1) écrivait: "Certains spécialistes pensent qu’il existe une norme québécoise différente de celle de la France; d'autres affirment que cette norme est au contraire identique au modèle international». Maurais (2008: 7-8) constatait aussi l'opposition entre partisans d'une norme exogène, d'un côté, et endogène, de l'autre. Plus récemment, Bigot et Papen (2013) soulignaient encore l'actualité du débat sur la norme du FQ, précisant «qu'une norme de prononciation du français québécois (et canadien en général) n’est pas encore entièrement déterminée" (Bigot et Papen 2013: 128). Dans un contexte où le FQ peine à être perçu unanimement par la société québécoise comme une variété pouvant produire un standard endogène et où les programmes d'enseignement de FLS, globalement, n'accordent pas au FQ la valeur de français de référence qu'ils attribuent au FF, il s'avère pertinent de s’interroger sur les attitudes des apprenants de FLS au Québec à l'égard du FQ et du FF.

\section{4. Études antérieures}

Quelques travaux consacrés aux perceptions et aux préférences d'immigrants, allophones ou apprenants de FLS, en ce qui a trait au FQ par rapport au FF, montrent comment s'articule cet enjeu. Les trois premières études (Saint-Laurent 2008; Maurais 2008; Calinon 2009) qui seront présentées ici n'examinent pas à proprement parler l'effet de la variation sociophonétique, mais s'interrogent sur les opinions d'immigrants, d'allophones et d'apprenants de FLS à propos de la langue française au Québec. La quatrième étude rapportée (Kircher 2012) se penche sur des réactions d'allophones de

4. À titre d'exemple, le cahier d'exercices Le français langue seconde par thèmes, niveau débutant, édité chez Chenelière

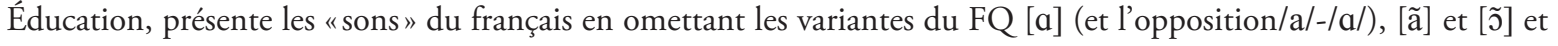

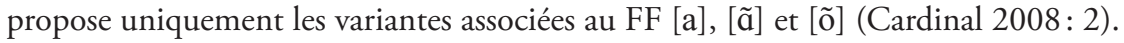

5. Variation «diatopique»: géographique, régionale; variation «diaphasique»: situationnelle, stylistique (Gadet 2007 : 23). 
Montréal envers l'accent du FQ et du FF, sans toutefois mettre de l'avant des variables sociophonétiques ni faire intervenir la variation stylistique.

Calinon (2009) propose une étude qualitative sur les opinions d'apprenants de FLS à Montréal, recueillies au moyen de groupes de discussion. Les participants de cette étude étaient des adultes d'origines diverses (Latino-Américains, Chinois, Roumains) fréquentant les programmes de francisation du Gouvernement du Québec dans différentes institutions à Montréal (Calinon 2009: 157-158). On y dénote une perception nettement plus négative que positive envers le $\mathrm{FQ}$, accompagnée d'une valorisation accrue pour le FF, vu comme la quasi seule forme normative, ou la «bonne» LC:

Pour nos témoins, le français hexagonal représente encore la norme standard (confortés par le matériel normatif et la langue enseignée dans les cours de francisation) alors que le français québécois porte les représentations négatives d'un dialecte oral qui ne lui permet pas d'accéder à un tel statut. (Calinon 2009: 258)

Selon l'auteure, la plupart des participants réalisent un amalgame entre les registres standard, familier et populaire du FQ, associant tout entière la variété du FQ à une langue uniquement non standard (Calinon 2009: 258). En outre, elle constate, tout comme Archambault et Corbeil (1982) ainsi que Bigot et Papen (2013: 128), le manque avéré de modèle linguistique québécois proposé par le matériel didactique et les programmes d'enseignement de FLS au Québec (Calinon 2009: 53, 258).

Maurais (2008), dans une vaste enquête téléphonique dont l'objectif était de "connaître l'état de l'opinion publique sur diverses thématiques liées à la norme linguistique: le français enseigné, la norme à privilégier dans les écoles" (Maurais 2008: 9-10), pose une question inspirée d'une enquête de Moreau (1990). Cette question est une mise en situation dans laquelle, devenu aphone un matin, le répondant devait choisir l'une de deux pilules: celle permettant de parler le FF ou celle permettant de parler le FQ. Les résultats montrent que les allophones de Montréal interrogés préfereraient parler dans une proportion de $63 \%$ la variété européenne, contre $37 \%$ pour la variété québécoise (Maurais 2008: 95).

Saint-Laurent (2008), dans une partie de son étude consacrée à la norme linguistique du Québec, rapporte que les participants, des immigrants consultés en groupes de discussion, préferent la norme issue du FF pour plusieurs raisons, d'entre lesquelles ressort la possibilité de «se faire comprendre dans toute la francophonie et non seulement au Québec» (Saint-Laurent 2008: 41). Parmi les participants de cette étude, tous âgés de 25 à 35 ans, se trouvait un sous-groupe d'immigrants établis au Québec depuis plus de trois ans, ainsi que des enfants de la loi $101^{6}$. Saint-Laurent (2008) indique que ces deux catégories de participants préferent un français à norme exogène, à cause de la perception qu'ils ont du FQ:

Le français québécois, c'est comme le niveau à ne pas atteindre. Le français international, c'est vraiment plus adapté et accepté dans les communautés [culturelles]. Dès que tu parles avec l'accent québécois, tu te le fais tellement dire. Je parle québécois, donc je parle mal. Si je parle vraiment québécois-région, on va me le dire que je parle mal. C'est laid, ça fait bûcheron, ça fait La p’itite vie, ça fait les Bougon, ça fait "pas de classe", ça fait mal instruit. C'est assimilé à trop pauvre. (Saint-Laurent 2008: 43)

6. Enfants de la loi 101 : «jeunes adultes âgés de 18 à 30 ans dont la langue maternelle n'est ni le français ni l'anglais et ayant terminé au moins quatre années d'études secondaires en français au Québec» (Saint-Laurent 2008: 13). 
La fusion produite ici par le participant entre variété (FQ) et niveau de langue (à ne pas atteindre) fait écho à l'amalgame aussi constaté par Calinon (2009). Saint-Laurent (2008) nuance cette dernière intervention en ajoutant que, pour une partie des participants de l'étude, recourir au français international tout comme au FQ «signifie [...] augmenter leurs chances de réussite professionnelle (d'où leur préférence pour le français international) [et] s'intégrer dans la société québécoise (d'où leur recours à la norme franco-québécoise et au français de niveau familier)» (Saint-Laurent 2008: 43).

Enfin, Kircher (2012), dans une enquête auprès d'allophones de la région de Montréal, âgés en moyenne de 18,3 ans et fréquentant le cégep en français, rapporte des attitudes plus négatives envers le FQ qu'envers le FF sur le plan du statut social associé aux langues. La valeur de langue standard, de langue de référence ou de "langue légitime» (Bourdieu 1982) est accordée au FF plutôt qu’au FQ. La méthode utilisée par Kircher (2012) est différente de celles empruntées dans les études présentées précédemment. Les participants n'ont ni répondu aux questions d'un sondage ni pris part à des discussions de groupe. Ils ont plutôt évalué des caractéristiques liées au statut social des locuteurs entendus ${ }^{7}$. L'auteure s'est servie de la technique du locuteur masqué (Lambert et al. 1960), une méthode indirecte par laquelle les participants évaluent des pistes sonores à l'aveugle. À quelques éléments près, c'est aussi la méthode qui a été utilisée dans la présente étude, qui fera l'objet d'une description détaillée dans la section 5. Ce qui peut dès lors être souligné est que, malgré des techniques d'enquête différentes, les attitudes et opinons des participants envers le FQ versus le FF observées dans les études précédentes suivent, globalement, une même direction.

Les travaux antérieurs ont clairement identifié certaines tendances, mais l'objectif de la présente étude n'est pas de confirmer ou d'infirmer une hypothèse particulière. Il s'agit plutôt d'observer autrement comment s'articulent les mêmes enjeux. De plus, le fait que les auteurs cités n'aient pas inclus la dimension stylistique de la variation dans leurs études me pousse à élargir la question. Les variantes linguistiques dites standards procédant notamment de l'évaluation sociale (Labov 1972), il m’apparait alors pertinent d'examiner aussi les attitudes d'apprenants de FLS à l'égard de ce type de variation ${ }^{8}$.

Deux questions liées aux constats que font les auteurs des travaux précédents sont visées dans la présente étude. Un des buts de l'étude est de vérifier si les styles FOR et INF, issus de chacune des variétés (FQ et FF), sont amalgamés par les apprenants de FLS au FQ ou au FF. La première question de recherche est formulée à partir de cet objectif: les styles FOR et INF issus de chacune des deux variétés dialectales sont-ils associés par les participants au Québec ou à la France? De plus, étant donné que les participants des études précédentes (Maurais 2008; Saint-Laurent 2008; Calinon 2009) déclaraient préférer parler un FF ou lui accordaient une plus grande valeur sociale (Kircher 2012), le second objectif est de connaitre la LC idéale des participants. La seconde question en découle: parmi les quatre possibilités suivantes, à savoir le FQ FOR et INF et le FF FOR et INF, laquelle est choisie comme LC par les participants?

\section{Méthode}

La «matched-guise technique» (Lambert et al. 1960), ou technique du locuteur masqué est une méthode d'enquête dont le principe repose sur une investigation indirecte des attitudes, c'est-à-dire,

7. Le questionnaire de Kircher (2012) comportait aussi des questions sur des trait de caractère reliés à la solidarité ressentie avec le locuteur/la langue. Cet aspect, n'étant pas de premier intérêt pour le sujet visé dans cet article, n'est pas abordé.

8. D'autant plus qu'est évoquée la notion de «niveau» de langue par un des participants (voir Saint-Laurent 2008). 
selon laquelle les participants ne doivent pas être informés des caractéristiques (p. ex. nationalité, langue maternelle) des locuteurs auxquels ils réagissent (Lambert et al. 1960, Kircher 2012). En comparaison avec une méthode directe (sondage, discussions), où les participants sont conscients des caractéristiques des objets jugés, la technique du locuteur masqué, en se soustrayant au biais de l'autocensure, permet d'avoir accès à des réponses réputées plus spontanées et valides sur les perceptions et opinions personnelles des participants (Lambert 1967; Laur 2008; Kircher 2012). Les stimuli auxquels les participants sont amenés à réagir sont faits d'enregistrements de mêmes individus dans des situations différentes. Les enquêtes menées avec cette technique (voir, entre autres, Lambert et al. 1960; d'Anglejan et Tucker 1973; Lappin 1982; Genessee et Holobow 1989; Kircher 2012; Laur 2014) se focalisent généralement sur les langues (français versus anglais) ou les variétés (FQ versus FF) et font appel à des locuteurs natifs bilingues ou bidialectaux lisant le même texte dans chacune de leurs deux langues ou variétés. Dans la présente étude, comme la variation stylistique est ciblée en plus de la variation dialectale, des locutrices du FQ et du FF ont été enregistrées dans des situations FOR et INF. Les participants n'étaient pas informés qu'ils entendaient des locutrices françaises et québécoises, ni qu'elles revenaient chacune deux fois dans la session d'écoute, soit dans les styles FOR et INF. Les différentes réactions des participants aux différentes pistes d'une même locutrice sont donc, en principe, induites par la variation linguistique (Lambert et al. 1960).

\subsection{Participants}

Si les chercheurs précédents ont interrogé des participants d'origines et de langues maternelles (L1) diverses, la présente étude est effectuée avec un groupe plus homogène. On sait que parmi les immigrants au Québec, «les personnes de langue maternelle latine [...] utilisent [...] plus le français comme langue publique prédominante» (Girard-Lamoureux 2004: 14). On pourrait donc s'attendre à une intégration plus facile et, éventuellement, une attitude plus positive envers le FQ de la part de certains groupes d'immigrants. Les seize hommes et vingt-sept femmes $(n=43)$ participant à la présente étude sont hispanophones et latino-américains. Ils sont tous apprenants de FLS à Montréal à des niveaux intermédiaire et avancé à l'École de langues de l'UQAM ou au programme du MIDI de l'Université de Montréal, du Cégep du Vieux-Montréal et de l'UQAM. ${ }^{9}$ La majorité d'entre eux étaient âgés, au moment de l'étude, de 30 à 39 ans $(\mathrm{n}=29)$, les autres se répartissant également dans les groupes d'âge suivant: 18 à 29 ans $(\mathrm{n}=7)$ et 40 à 50 ans $(\mathrm{n}=7)$. Le pays d'origine le plus fréquemment représenté est la Colombie $(\mathrm{n}=21)$, suivi du Venezuela $(\mathrm{n}=7)$ et du Mexique $(\mathrm{n}=7)$; viennent ensuite Cuba $(\mathrm{n}=3)$, le Pérou $(\mathrm{n}=2)$, l'Uruguay $(\mathrm{n}=1)$, le Nicaragua $(\mathrm{n}=1)$ et l'Équateur $(\mathrm{n}=1)$.

\subsection{Stimuli}

Dans cette section, seront décrites les locutrices et l'élaboration des stimuli, ainsi que la variation dialectale et stylistique présente dans les pistes sonores soumises à l'écoute des participants.

9. Programme du MIDI : Programme du ministère de l'Immigration, de la diversité et de l'inclusion. Pour les compétences des apprenants de niveaux intermédiaire et avancé, voir la banque d'exercices leur étant destinés en ligne sur le site du MIDI (consultée le 15 mars 2018) : http:/www.micc-francisation.gouv.qc.ca/site/skin/htm/default.htm. En outre, même si l'expérimentation ne vise pas la compréhension orale, des participants de niveau débutant n'ont pas été sollicités à cause de la possibilité que leur compétence en FLS interfère avec la tâche demandée. 


\subsubsection{Locutrices}

Les huit pistes sonores qui constituent les stimuli ont été enregistrées par quatre locutrices natives du français: deux Québécoises, nées ou ayant vécu pendant l'enfance ou l'adolescence à Montréal, et deux Françaises, nées ou ayant vécu pendant l'enfance ou l'adolescence à Paris. Le tableau 1 propose la synthèse des caractéristiques des locutrices et les identifie avec des pseudonymes. On sait que les différences de sexe des locuteurs entendus conduisent à des différences dans les réactions des auditeurs-évaluateurs (Labov 2006; Laur 2008, 2014). Par conséquent, des individus du même sexe ont été choisis (ici, des locutrices) afin d'exclure la variance qui pourrait être induite par la préférence des participants pour un sexe ou l'autre, lors de l'écoute des stimuli (Labov 2006; Thomas 2002). De plus, afin de s'assurer d'une bonne flexibilité sur le plan stylistique, les locutrices sont toutes issues du milieu des arts de la scène (chanson, musique, cirque, théâtre).

Tableau 1: Description des locutrices

\begin{tabular}{lccc}
\hline \multicolumn{1}{c}{ Locutrices } & Lieu de naissance & Années vécues à Paris/Montréal & Âge \\
\hline MARIE & Reims & $15(\mathrm{PAR})$ & 32 \\
AMÉLIE & Paris & $24(\mathrm{PAR})$ & 28 \\
MIREILLE & Laval & $11(\mathrm{MTL})$ & 29 \\
CATHERINE & Montréal & $23(\mathrm{MTL})$ & 37 \\
\hline
\end{tabular}

\subsection{2 Élaboration et enregistrements des stimuli}

Afin d'obtenir des pistes sonores INF et FOR contenant exactement les mêmes mots, suites de mots et phrases, et d'isoler par le fait même la variation phonétique, un test expressif (pour la version INF) et une lecture préparée (pour la version FOR) ont été créés à partir des mêmes phrases cibles. Ces stimuli ont été conçus afin de favoriser l'occurrence de variables sociophonétiques qu'on retrouve sur les plans dialectal et stylistique, en fonction de la plus ou moins grande formalité de la situation ${ }^{10}$. Pour ce faire, 17 phrases cibles contenant des variables ciblées ont été créées. Ces variables ont des variantes associées au FQ, au FF, ou encore aux styles FOR et INF. Les phrases cibles contenues dans les stimuli sont: C'est une petite fille qui boit du lait. C'est une petite fille qui se regarde dans le miroir. C'est un petit garçon qui regarde une maison en bois. C'est une petite fille qui fait des bulles. C'est une petite fille qui mange des pâtes. C'est un chat qui se lèche la patte. C'est une guêpe. C'est un piquet de clôture. C'est une ville. C'est un village. C'est du pain brun. C'est une femme qui regarde une voiture. Trois arbres. Quatre zèbres. Mille mètres égalent un kilomètre. C'est une serveuse. Quatre serveurs regardent trois serveuses.

La première des trois étapes de l'enregistrement des stimuli a consisté en une entrevue sociolinguistique de trente minutes sous forme de conversation à trois: deux locutrices de la même variété dialectale ayant des liens d'amitié entre elles, pour favoriser la production de variantes INF (Feagin 2002), et l'intervieweuse. Les sujets abordés étaient le quotidien et les souvenirs d'enfance. Le but de cette entrée en matière n'était que de détendre l'atmosphère et de permettre aux locutrices de parler

10. La formalité de la situation a été divisée de façon binaire, soit FOR versus INF, pour les besoins de la recherche, sans pour autant prétendre à une division strictement binaire dans les observations qu'on peut faire des différents styles que peut adopter un locuteur. 
sans qu'une attention particulière ne soit portée à leur langue. En outre, ce préambule permettait de se familiariser avec la présence du mini-magnétophone (Olympius WS-811). Les enregistrements de cette première étape n’ont pas été utilisés pour les stimuli.

Pour la deuxième étape, la production de variantes INF, un test de type expressif a été proposé aux locutrices. À la manière d'un jeu (rébus), dont le motif prétendu était une recherche sur les représentations iconiques selon la culture d'origine, les locutrices ont regardé les images et les animations qui visaient la production des phrases cibles mentionnées plus haut. Il leur était simplement demandé de dire ce qu'elles y voyaient. Les questions posées par l'intervieweuse étaient C'est quoi? ou C'est quoi, ça?. Pour s'assurer que les locutrices produisent les phrases cibles, conçues pour être identiques en situations INF et FOR (sur le plan syntaxique et lexical), certains items cibles ont d'abord été mis en place, par exemple, en proposant une image montrant, sur le même écran, une femme, une fille, une petite fille et un bébé. Les locutrices devaient alors nommer ce qu'elles voyaient. Les réponses attendues étaient (une) fermme, (une) fille, (une) petite fille et (un) bébé. Une fois que la locutrice donnait la cible (une) petite fille, elle était informée qu'on allait se concentrer sur ce personnage dans les prochaines images. Ainsi, lorsqu'on proposait, par la suite, l'image d'une petite fille se regardant dans un miroir ou soufflant dans un dispositif permettant de faire des bulles de savon, les phrases cibles C'est une petite fille qui se regarde dans le miroir et C'est une petite fille qui fait des bulles étaient produites par les locutrices après la question C'est quoi, (ça)? de l'intervieweuse. La même situation a été répétée avec une image sur laquelle figuraient un homme, un garçon, un petit garçon et un bébé. Tant que la locutrice ne prononçait pas la phrase voulue, l'intervieweuse, qui ne prononçait jamais les cibles, procédait par questions et discussion sur l'image afin de la lui faire produire. À l'exception de quelques images qui ont été moins bien déchiffrées par les locutrices, le test expressif était suffisamment bien monté (et auparavant testé) pour que les phrases cibles telles que conçues soient produites spontanément par les locutrices ${ }^{11}$. La rencontre, dans les deux premières parties, visait une langue spontanée; le véritable motif de la recherche n'était pas dévoilé.

Pour la troisième étape, une version écrite de ces mêmes phrases cibles, destinée à être lue, a été présentée aux locutrices. La consigne qui accompagnait la tâche était de préparer la lecture en vue d'une audition pour devenir lectrice de nouvelles à la radio d'État. Par rapport au test expressif, conduit dans une atmosphère détendue, spontanée et conviviale, cette lecture avait comme fonction d'augmenter la formalité de la situation. Pour avoir accès aux variantes normatives des locutrices, par le biais d'une lecture dans un contexte très FOR (Labov 1972: 79-80), la stratégie était de faire en sorte que les locutrices sentent la nécessité de surveiller leur langue et varient leur prononciation, stylistiquement, par rapport à leur production spontanée lors du test expressif. Ces enregistrements ont été montés avec le logiciel Audacity (Mazzoni et Dannenbergen 1999) en huit pistes sonores destinées à l'écoute des participants, soit une piste FOR et une piste INF pour chaque locutrice du FQ et pour chaque locutrice du FF. La longueur des pistes sonores était d'environ 30 secondes chacune ${ }^{12}$.

11. Lorsque des images ont donné lieu à des hésitations ou des phrases non attendues, ces phrases n’ont pas été retenues dans les stimuli.

12. Les enregistrements de départ contenaient cinquante phrases cibles, dont les 17 présentées dans cet article et retenues pour les stimuli, choisies soit parce que leur production avait été facile et spontanée en regard des phrases attendues lors du test expressif, ou parce qu'elles contenaient plus de variables que les autres, ce qui rendait les contrastes dialectaux et stylistiques plus évidents. Le montage a été opéré pour sélectionner les 17 mêmes phrases pour chaque locutrice et chaque style. 


\subsubsection{Variation sociophonétique dans les stimuli}

Au moment de la conception des stimuli, les variables ont été choisies en fonction du caractère contrastant qu'elles présentent entre variétés dialectales. En outre, certaines de ces variables généralement associées au FQ peuvent aussi être attestées comme des variables stylistiques au Québec; elles seront identifiées, le cas échéant. Sur l'axe dialectal, les variables ciblées et variantes obtenues dans les enregistrements, présentées au tableau 2 , sont ${ }^{13}$ :

- des oppositions phonologiques telles que et /a/—/a/ (p. ex. patte/pâte), /wa/— /wa/ (p. ex. (je) bois/(le) bois) et $/ \tilde{\varepsilon} /$ - / $\tilde{\mathfrak{e}} /$ (p. ex. brin/brun), maintenues en FQ versus neutralisées en FF (Dumas 1987; Ostiguy et Tousignant 2008; Hansen et Julliard 2011; Hansen, 2012) ${ }^{14}$;

- la réalisation de /a/ et /wa/ en finale de mot (p. ex. chat, le bois), qui alterne entre [a], [0] et possiblement [a] (hypercorrection) en FQ (Dumas 1987; Ostigy et Tousigant 2008; Paradis et Dolbec 2008; Brasseur 2009; Côté 2012), versus les réalisations antérieures [a] et [wa] en FF (Hansen 2012; Lyche et al. 2012) ${ }^{15}$;

- l'affrication ou assibilation (FQ), versus la non-affrication (FF), des consonnes (C) /t/ et /d/ devant les voyelles (V) hautes antérieures /i, y/, p. ex. petit (Dumas 1987; Cox 1998; Ostiguy et Tousignant 2008; Paradis et Dolbec 2009; Brasseur 2009; Detey et al. 2010; Friesner 2010 ; Lyche et al. 2012; Blondeau et Friesner 2014; Côté 2014) ${ }^{16}$;

- le relâchement (FQ) versus la tension (FF) des voyelles hautes /i, y/ en syllabe finale fermée, p. ex. ville (Dumas 1987; Ostigy et Tousigant 2008; Paradis et Dolbec 2008; Brasseur 2009; Detey et al. 2010 ; Friesner 2010) ${ }^{17}$;

13. Certaines de ces variables, identifiées comme appartenant au FQ, comme l'affrication et le relâchement des V hautes, apparaissent aussi dans le français parlé ailleurs au Canada (Alain 1989: 57; Mougeon et Beniak 1994; Walker 2005: 191). Il n'est pas question ici de décrire «le» FQ en excluant les traits qui sont partagés ou exclus par une communauté plus ou moins grande, mais de se concentrer sur ce qui est rapporté, dans la littérature, comme typique, voire stéréotypé (Dumas 1987: 7; Brasseur 2009; Friesner 2010). De la même manière, la bannière FF pour le français de France n'est pas une tentative de décrire toutes les variétés de français parlées en France, ni même à Paris, comme si elles y étaient homogènes (Walter 1982).

14. L'opposition /e/ vs /E/, maintenue en FQ, était aussi ciblée dans l'item piquet, mais les locutrices ont toutes produit la même voyelle, à savoir plutôt $[\varepsilon]$ que [e]. C’est pourquoi cette variable n’a pas été retenue.

15. La diphtongue/wa/-/wa/connait aussi des variantes en FQ comme « [we], [we], [we:], [wa], [wa], [wo]» etc. (Paradis et Dolbec 2008), mais seules les variantes effectivement produites par les locutrices de l'étude sont présentées dans le texte, faute d'espace. En outre, ces variables peuvent aussi être diphtonguées (Côté 2012), ce qui n’a pas été le cas dans la présente étude. Il est à noter que les réalisations fermées en [0] peuvent être sujettes à des jugements sociaux négatifs (Paradis et Dolbec 2008; Côté 2012). Tout comme pour /a/, la variable peut, en situation FOR, être réalisée $[\mathrm{wa}]$, voire [wa], mais cette dernière est vue comme une hypercorrection (Dumas 1987; Ostiguy et Tousignant 2008).

16. L'affrication n'est généralement pas stigmatisée au Québec, tant elle est courante (Ostiguy et Tousignant 2008; Paradis et Dolbec 2008). Cependant, Dumas (1987: 9) note que dans les cas "phonétiquement très appuyés», elle est considérée comme inélégante, voire vulgaire.

17. Le relâchement s'applique aussi normalement à /u/, mais les stimuli ne contiennent aucune occurrence de cette voyelle. On parle en général du relâchement comme d'une variante non stigmatisée au Québec (Ostiguy et Tousignant 2008; Paradis et Dolbec 2008). Néanmoins, selon Paradis et Dolbec (2008) ainsi que Dumas (1987: 95), la centralisation peut être amoindrie en situation formelle, ou même absente des communications à caractère très formel. 
- la réalisation diphtonguée (FQ) versus monophtonguée (FF) des voyelles longues ou devant une consonne allongeante en syllabe finale fermée, p. ex. serveuse, guêpe, pâte (Dumas 1987; Ostigy et Tousigant 2008; Paradis et Dolbec 2008; Côté 2012) ${ }^{18}$;

- le timbre de la version longue de/ع/, réalisé [3] en FQ, versus [ع] en FF, p. ex. guêpe (Santerre1974; Morin 2009; Côté 2012) ${ }^{19}$;

- la réalisation antérieure [ã] $(\mathrm{FQ})$ versus postériorieure $[\widetilde{a}](\mathrm{FF})$ de la voyelle nasale / $\tilde{\mathrm{A}} /$, p. ex. mange (Ostiguy et Tousignant 2008; Paradis et Dolbec 2008; Brasseur 2009);

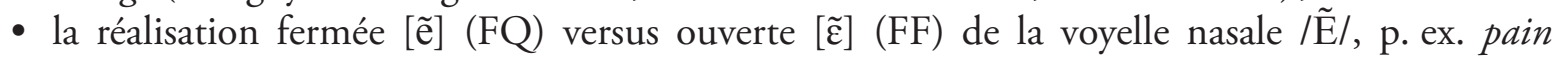
(Ostiguy et Tousignant 2008);

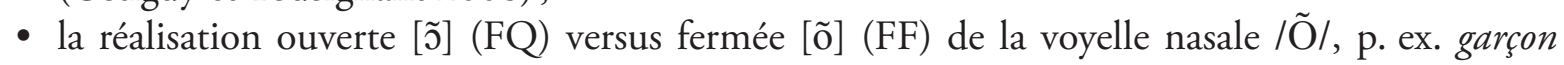
(Ostiguy et Tousignant 2008).

Tableau 2: Variables sur l'axe dialectal présentes dans les stimuli

\begin{tabular}{|c|c|c|c|c|c|}
\hline Variables & $\begin{array}{c}\text { Variantes } \\
\text { FQ } \\
\end{array}$ & $\begin{array}{c}\text { Variantes } \\
\text { FF } \\
\end{array}$ & Ex. dans stimuli & Réalisations FQ & $\begin{array}{c}\text { Réalisations } \\
\text { FF }\end{array}$ \\
\hline oppositions & maintenues & neutralisées & $\begin{array}{c}\text { pâte (/patte) } \\
\text { bois (/je bois) } \\
\text { brun (/brin) }\end{array}$ & 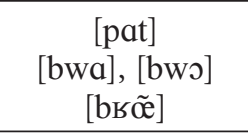 & $\begin{array}{c}\text { [pat] } \\
{[\text { bwa }]} \\
{[\text { bь } \tilde{\varepsilon}]}\end{array}$ \\
\hline /a/, /wa/ & $\begin{array}{c}\text { [a] (post.) } \\
\text { [o] (fermée) }\end{array}$ & [a] (ant.) & $\begin{array}{c}\text { chat } \\
\text { (le) bois }\end{array}$ & $\begin{array}{c}{\left[\int a\right],\left[\int \rho\right]} \\
{[b w a],[b w o]}\end{array}$ & $\begin{array}{c}{\left[\int \mathrm{a}\right]} \\
{[\mathrm{bwa}]}\end{array}$ \\
\hline assibilation /t, d/ & oui & non & petit & {$\left[\operatorname{pət}^{\mathrm{s}} \mathrm{i}\right]$} & [pəti] \\
\hline V hautes CVC & $\begin{array}{l}\text { centralisées } \\
\text { (relâchées) }\end{array}$ & tendues & ville & {$[\mathrm{VIl}]$} & [vil] \\
\hline timbre / $\varepsilon /$ long & [3] & {$[\varepsilon]$} & guêpe & {$[\mathrm{g} 3 \mathrm{p}]^{*}$} & [gep] \\
\hline stabilité V longues & diphtongue & monophtongue & $\begin{array}{c}\text { serveuse } \\
\text { pâte } \\
\text { guêpe }\end{array}$ & $\begin{array}{c}{\left[\operatorname{sebVø}^{\mathrm{y}} \mathrm{z}\right]} \\
{\left[\mathrm{pa}^{\mathrm{u}} \mathrm{t}\right]} \\
{\left[\mathrm{g}^{\mathrm{a}} 3 \mathrm{p}\right],\left[\mathrm{g}^{\mathrm{i}} \mathrm{p}\right]}\end{array}$ & $\begin{array}{c}\text { [sebVøz] } \\
\text { [pat] } \\
\text { [gep] }\end{array}$ \\
\hline $\mathrm{V}$ nasales & $\begin{array}{c}\text { [ãa (ant.) } \\
{[\tilde{\mathrm{e}}] \text { (fermée) }} \\
{[\tilde{\mathrm{o}}] \text { (ouverte) }}\end{array}$ & $\begin{array}{c}{[\tilde{\mathrm{a}}] \text { (post.) }} \\
{[\tilde{\varepsilon}] \text { (ouverte) }} \\
{[\tilde{\mathrm{o}}] \text { (fermée) }}\end{array}$ & $\begin{array}{l}\text { mange } \\
\text { pain } \\
\text { garçon }\end{array}$ & $\begin{array}{c}{[\mathrm{mãz}]} \\
{[\mathrm{pe \tilde {e }}]} \\
{[\text { gass }]}\end{array}$ & $\begin{array}{c}{[\mathrm{ma} z]} \\
{[\mathrm{p} \tilde{\varepsilon}]} \\
{[\text { gassõ] }}\end{array}$ \\
\hline
\end{tabular}

* Les transcriptions données en exemple ne tiennent compte que de la variable qu'elles illustrent. La durée des voyelles n'est donc pas indiquée, ni la possible diphtongaison des voyelles longues pour les variables portant sur le timbre (p. ex. guêpe pour le timbre de /ع/ long et mange pour le timbre des voyelles nasales).

Sur l'axe diaphasique, les variables ciblées, présentées au tableau 3, ont été choisies en fonction du fait qu'elles sont considérées comme stylistiques pour les deux variétés dialectales.

18. Le phénomène peut aussi être considéré comme une variante stylistique (Paradis et Dolbec 2008; Detey et al. 2010; Côté 2012) dans la mesure où une diphtongaison dite "forte» peut entrainer une évaluation sociale négative au Québec et diminue dans les situations formelles (Paradis et Dolbec 2008).

19. Le symbole [3], proposé par Santerre (1974) et repris, entre autres, par Morin et al. (1990) et Côté (2012) est utilisé pour préciser le timbre de la voyelle de guêpe (ou fête vs faite) en FQ, différent du timbre [ع] en FF. Santerre (1974) montre des différences sur les plans acoustiques, articulatoires et perceptuels. Morin (2009) propose aussi [æ]. Merci à M.-H. Côté de m'avoir signalé cette variante. 
Tableau 3: Variables sur l'axe stylistique présentes dans les stimuli

\begin{tabular}{|c|c|c|c|c|c|}
\hline Variables & $\begin{array}{c}\text { Exemples dans } \\
\text { stimuli }\end{array}$ & Variantes FOR & $\begin{array}{l}\text { Variantes } \\
\text { INF }\end{array}$ & $\begin{array}{l}\text { Situation } \\
\text { FOR }\end{array}$ & $\begin{array}{l}\text { Situation } \\
\text { INF }\end{array}$ \\
\hline liaison & c'est un(e) & $\begin{array}{l}{[\operatorname{set} \tilde{\tilde{e}}]} \\
{[\operatorname{set} \tilde{\varepsilon}]}\end{array}$ & $\begin{array}{c}{[\mathrm{se} \tilde{\tilde{Q}}]} \\
{[\mathrm{se} \tilde{\varepsilon}]}\end{array}$ & + réalisation & - réalisation \\
\hline gr. cons. finaux & $\begin{array}{l}\text { arbre } \\
\text { quatre }\end{array}$ & $\begin{array}{l}\text { [авbь }] \\
{[\text { kats] }}\end{array}$ & $\begin{array}{l}\text { [asb] } \\
{[\mathrm{kat}]}\end{array}$ & - réduction & + réduction \\
\hline schwa & regarde & [вәgавd(ə)] & [вgавd] & + présence & + absence \\
\hline
\end{tabular}

Les variables et variantes stylistiques ciblées et obtenues pour les deux variétés dialectales dans les stimuli sont:

- la réalisation (FOR) ou la non-réalisation (INF) de la consonne /t/ dans le site de liaison non catégorique c'est un/e ${ }^{20}$;

- la réduction (INF) ou prononciation complète (FOR) des groupes consonantiques finaux (Pupier et Drapeau 1973; Gadet 2007; Ostiguy et Tousignant 2008, Côté 2004);

- l'absence (INF) ou la présence (FOR) du schwa (Ostiguy et Tousignant 2008; Hansen 2012).

\subsubsection{Comportement des locutrices en fonction du style}

Les résultats d'un dénombrement des variantes stylistiques par locutrice et par situation (FOR, INF) pour les variables communes aux deux variétés dialectales sont présentés au tableau 4. Ces observations ont été produites sur une base impressionniste.

Tableau 4: Variation stylistique par locutrice

\begin{tabular}{|c|c|c|c|c|c|c|c|c|}
\hline \multirow[b]{2}{*}{ Variables } & \multicolumn{4}{|c|}{ INF } & \multicolumn{4}{|c|}{ FOR } \\
\hline & $\begin{array}{c}\text { Marie } \\
\text { FF }\end{array}$ & $\begin{array}{l}\text { Amélie } \\
\text { FF }\end{array}$ & $\begin{array}{c}\text { Mireille } \\
\text { FQ }\end{array}$ & $\begin{array}{c}\text { Cath. } \\
\text { FQ }\end{array}$ & $\begin{array}{c}\text { Marie } \\
\text { FF }\end{array}$ & $\begin{array}{c}\text { Amélie } \\
\text { FF }\end{array}$ & $\begin{array}{c}\text { Mireille } \\
\text { FQ }\end{array}$ & $\begin{array}{c}\text { Cath. } \\
\text { FQ }\end{array}$ \\
\hline $\begin{array}{l}\text { liaison } \\
\text { c'est unte }\end{array}$ & $10 / 12$ & $6 / 12$ & $6 / 12$ & $11 / 12$ & $12 / 12$ & $12 / 12$ & $12 / 12$ & $12 / 12$ \\
\hline réduc. gr. cons. & $2 / 6$ & $0 / 6$ & $1 / 6$ & $0 / 6$ & $0 / 6$ & $0 / 6$ & $1 / 6$ & $0 / 6$ \\
\hline schwa & $9 / 16$ & $6 / 16$ & $4 / 16$ & $5 / 16$ & $14 / 16$ & $15 / 16$ & $5 / 16$ & $13 / 16$ \\
\hline
\end{tabular}

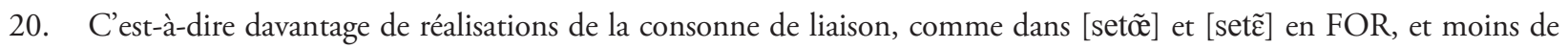

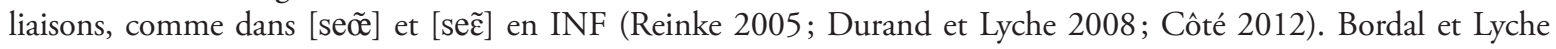
(2008) mentionnent pour Paris des taux de liaison après ( $c^{\prime}$ est qui passent de $92 \%$ en FOR (lecture) à $35 \%$ en conversation. Côté (2012) précise que, selon les données du projet Phonologie du Français Contemporain (PFC), pour Trois-Rivières, au Québec, les taux de liaison avec (c')est sont plus élevés en conversation (87 \%) que dans les autres variétés du français, y compris le FF (Côté 2012: 266). Néanmoins, l'auteure précise aussi que pour toutes les variétés du français, incluant le FQ, les taux de liaison sont généralement plus élevés en style plus FOR (Côté 2012: 267). Reinke 2005 rapporte un taux d'omission des liaisons facultatives de 33,2 \% (réalisation de 66,8\%) par les animateurs et lecteurs de nouvelles dans des émissions de télévision et de radio québécoise à caractère FOR (Reinke 2005: 34-35). Les locutrices de la présente étude ont eu des comportements plus et moins alignés avec les observations des chercheurs mentionnés, avec des taux de $50 \%$ (2 locutrices), $83 \%$ et $91 \%$ en conversation et de $100 \%$ en lecture. Les différences entre les observations de la présente étude et les précédentes peuvent être dues à la nature de la liaison c'est un $(e)$ et au faible nombre de locutrices $(\mathrm{n}=4)$, limite imposée par la nature des études sur les attitudes linguistiques. 
Pour la liaison de c'est un/e, en style INF, Amélie (FF) et Mireille (FQ) ont produit $50 \%$ (6/12) des liaisons, alors que Marie (FF) et Catherine (FQ) ont produit respectivement dix (83\%) et onze (91\%) liaisons sur douze contextes possibles. En style FOR (lecture), les quatre locutrices ont produit $100 \%$ des liaisons de c'est un/e. En situation INF, le schwa a été prononcé neuf fois par Marie (FF) et six fois par Amélie (FF), ainsi qu'à quatre reprises pour Mireille (FQ) et cinq reprises pour Catherine (FQ), sur un potentiel de seize occurrences. En situation FOR, la prononciation du schwa a eu lieu quatorze fois pour Marie (FF) et quinze fois pour Amélie (FF). Alors que Mireille (FQ) a effectué très peu de changement par rapport à sa piste INF et a produit cinq schwas en situation FOR, Catherine (FQ) en a produit treize. Pour la réduction des groupes de consonnes finales, en situation INF, Marie (FF) produit deux réductions et Mireille (FQ), une, sur six contextes. En situation FOR, une seule réduction a eu lieu pour Mireille (FQ) ${ }^{21}$.

Sur les variables mentionnées, la variation stylistique entre les Françaises et les Québécoises reste comparable, à l'exception du schwa. En effet, Mireille (FQ), qui l'a produit moins de fois que les autres locutrices en situation INF, maintient ce comportement en situation FOR, alors que les autres locutrices ont considérablement augmenté leur nombre d'occurrences de schwas en situation FOR. Aussi, Amélie (FF) est la locutrice qui a montré en situation INF le comportement le moins normatif des deux Françaises (taux de liaison et de réalisation du schwa).

La variation stylistique produite par les locutrices du FQ sur des variantes généralement associées au FQ est présentée dans le tableau 5. Puisque certains comportements perceptibles à l'écoute demeurent difficiles à évaluer de manière précise à l'oreille, deux variables ont fait l'objet d'analyses acoustiques: le relâchement des voyelles hautes en syllabe fermée et la durée des affrications (bruit de la consonne affriquée). La diphtongaison et /(w)a/ en finale absolue ont été analysés uniquement de manière impressionniste.

Pour le relâchement des voyelles hautes, les valeurs des deux premiers formants F1 et F2 ont été mesurées à l'aide d'une analyse LPC (Linear Predictive Coding) intégrée dans le programme Praat (Boersma et Weenink 2016) et les trapèzes vocaliques ont été analysés. Le but était de vérifier si la distance entre les voyelles hautes [i, y] en syllabe fermée et le [a] de patte était plus petite en contexte INF que FOR, ou, autrement dit, si ces voyelles étaient moins centralisées en situation FOR. Une comparaison a aussi été effectuée entre les distances des voyelles hautes antérieures en syllabe fermée et ouverte et une occurrence de [a], à l'opposé du trapèze vocalique. On souhaitait ainsi voir si la distance des occurrences de [i, y] en syllabe fermée des situations FOR égalait ou, éventuellement, dépassait la distance moyenne de ces mêmes voyelles lorsqu'elles sont non relâchées, en syllabe ouverte. Dans le tableau 5, la variable est qualifiée de plus (+) relâchée lorsque la locutrice a globalement produit des voyelles hautes antérieures en syllabe fermée dont la distance avec [a] était plus petite que celle de ses voyelles hautes en syllabe ouverte. La variable est qualifiée de moins (-) relâchée lorsque la locutrice a globalement produit ses voyelles hautes antérieures en syllabe fermée dans une distance avec [a] relativement égale ou supérieure à celle de ses voyelles hautes en syllabe ouverte, et supérieure à la distance observée pour la situation INF. La variable est finalement qualifiée de beaucoup moins (- -) relâchée

21. Le fait que les locutrices aient peu réduit leurs groupes de consonnes finales en situation INF est possiblement attribuable à plusieurs facteurs, entre autres la prosodie et la position des groupes de consonnes dans la phrase (en de fin de syntagme nominal avant un syntagme verbal et en déterminant numéral), la nature de la tâche (devoir dire ce qui est vu, notamment le chiffre " 4 ») et, finalement, un effet d'amorce et d'entrainement, les items contenant la variable se suivant de près dans le test expressif. Le caractère représentatif des locutrices par rapport aux variétés du FQ comme du FF n'est pas revendiqué ici étant donné leur nombre (4). 
lorsque la locutrice a globalement produit ses voyelles hautes antérieures en syllabe fermée dans une distance avec [a] relativement égale ou supérieure à celle de ses voyelles hautes en syllabe ouverte, et allant jusqu’à doubler cette distance dans la situation FOR par rapport à la situation INF.

Tableau 5: Variation stylistique sur des variantes de FQ

\begin{tabular}{lccccc}
\hline \multirow{2}{*}{ Variables } & \multicolumn{2}{c}{ INF } & \multicolumn{2}{c}{ FOR } \\
\cline { 2 - 5 } & Mireille & Cath. & Mireille & FQ & $\begin{array}{c}\text { Cath. } \\
\text { FQ }\end{array}$ \\
\cline { 2 - 6 } & + & FQ & + & - & - \\
\hline rel. V hautes & $=$ & + & $1 / 7$ & - \\
\hline durée assibilation & $3 / 7$ & $5 / 7$ & {$[\mathrm{a}][\mathrm{a}]$} & {$[\mathrm{a}]$} \\
\hline diphtongaison & {$[\mathrm{o}],[\mathrm{a}]$} & {$[\mathrm{0}]$} & &
\end{tabular}

Les mesures ont en fait confirmé et détaillé ce qui était déjà repéré à l'écoute. Les analyses acoustiques ont permis de voir que Mireille a moins centralisé ses voyelles hautes antérieures en syllabe fermée dans la situation FOR pour onze des treize occurrences de /y/ et pour l'ensemble des dix occurrences de $[\mathrm{i}]^{22}$. Elle a produit en situation FOR des voyelles aussi tendues et relativement plus tendues, pour cinq occurrences de [i], que la moyenne de ses [i] en syllabe ouverte ${ }^{23}$. Catherine a montré encore plus de contrôle. Elle a réalisé des variantes moins centralisées en situation FOR qu'en situation INF pour l'ensemble des treize occurrences de [y], dont quatre occurrences étaient plus tendues que la moyenne de ses voyelles hautes en syllabe ouverte ${ }^{24}$. De même pour l'ensemble des dix occurrences de [i], dont quatre occurrences étaient aussi ou plus tendues que la moyenne des [i] en syllabe ouverte ${ }^{25}$. En outre, en situation FOR, par rapport à la situation INF, Catherine a doublé la distance de certains de ses /i/ en syllabe fermée par rapport à son [a] ${ }^{26}$.

Pour la durée des assibilations, un calcul de la durée du bruit suivant l'explosion des consonnes [t, d] avant la production des voyelles hautes antérieures a été effectué, toujours avec Praat (Boersma et Weenink 2016). Cela a permis de vérifier si les durées des assibilations sont réduites par les locutrices dans les situations FOR par rapport aux situations INF, suivant ce qui a été remarqué de manière impressionniste. Les durées ont été comparées entre les styles FOR et INF pour les mêmes items (par exemple petite) pour chaque locutrice. Le tableau 5 montre si les durées ont été comparables $(=)$ entre les styles, plus (+) longues que pour l'autre style, ou plus courtes (-). En situation FOR par rapport à la situation INF, Mireille n’a pas réduit de façon régulière ses assibilations, qui demeurent globalement comparables en ce qui a trait à la durée ${ }^{27}$. Par contre, Catherine a réduit systématiquement, en

22. [y] : distance moyenne en INF: $664 \mathrm{~Hz}$ et en FOR: $877 \mathrm{~Hz}$; [i] : distance moyenne en INF: $847 \mathrm{~Hz}$ et en FOR: $1083 \mathrm{~Hz}$.

23. fille x 3: $1136 \mathrm{~Hz}, 1194 \mathrm{~Hz}, 1231 \mathrm{~Hz}$; ville: $1143 \mathrm{~Hz}$; mille: $1287 \mathrm{~Hz}$; moyenne en syllabe CV: $1133 \mathrm{~Hz}$.

24. [y] : distance moyenne en INF: $434 \mathrm{~Hz}$ et en FOR: $505 \mathrm{~Hz}$; une x 2: $576 \mathrm{~Hz}, 529 \mathrm{~Hz}$; bulle: $538 \mathrm{~Hz}$; clôture: $614 \mathrm{~Hz}$; moyenne en syllabe CV: $519 \mathrm{~Hz}$.

25. fille x 3: $1218 \mathrm{~Hz}, 1212 \mathrm{~Hz}, 1157 \mathrm{~Hz}$; petite: $1247 \mathrm{~Hz}$; moyenne en syllabe CV: $1136 \mathrm{~Hz}$.

26. Distance moyenne en INF: $544 \mathrm{~Hz}$ et en FOR: $1035 \mathrm{~Hz}$.

27. Pourcentage moyen de la durée de l'assibilation sur la durée totale de la consonne pour [dy]: INF 62,2 \%; FOR $61,6 \%$; [ti] : INF 66,1\%; FOR 65,7\%; INF [ty] : 74,1\%; FOR 70,9\%. 
situation FOR, ses durées d'assibilation, retranchant jusqu'à $20 \%$ de la durée pour le contexte [ti], très présent dans les stimuli, par exemple dans les items petit et petite ${ }^{28}$.

Pour les diphtongaisons qu'on peut qualifier de franches à l'oreille, Mireille en a produit trois en situation INF, soit sur les items guêpe, mètre et kilomètre. Catherine en a produit cinq, sur les items guêpe, mètre, kilomètre et serveuse (2 occurrences), sur sept contextes possibles (pâtes et serveur non diphtongués). En situation FOR, les deux locutrices ont produit une seule diphtongaison sur l'item guêpe. Finalement, pour la réalisation de /a/ et /wa/ en finale absolue, Mireille a produit les variantes fermées [0] et postérieures [a] en situation INF, qu'elle a corrigées, respectivement, en variantes postérieures [a] et antérieures [a] pour les items bois et chat dans la piste FOR. Catherine a produit la variante fermée [0] en situation INF et a utilisé la variante postérieure [a] pour bois en situation FOR.

Il est à noter que les Québécoises en situation FOR ont à la fois tendu vers un modèle de FF, international ou exogène, en vertu du contrôle exercé sur le relâchement des voyelles hautes et l'assibilation (voir Dumas 1987; Ostiguy et Tousignant 2008), et conservé des traits dits caractéristiques du FQ, comme la diphtongaison et les finales en [a]. Par ailleurs, Catherine a montré un comportement plus normatif que Mireille sur trois variables, si on considère les variables dialectales et stylistiques, soit l'assibilation, le relâchement des voyelles hautes et le schwa.

\subsection{Collecte des données évaluatives}

Dans un local de classe muni d'ordinateurs, les participants ont écouté, à l'aide de casques d'écoute, les pistes sonores les unes après les autres, en répondant après chaque piste aux mêmes questions, présentées à l'écran ${ }^{29}$. Les questions traitées dans la présente étude comportent, pour l'une, deux choix de réponse, et pour l'autre, quatre choix de réponse de type échelle de Likert. Ces questions étaient rédigées comme suit: A) Selon vous, la personne que vous entendez provient: 1) de France; 2) du Québec; et B) Cette personne parle le français que je veux apprendre. 1) non, pas du tout; 2) non, pas vraiment; 3) oui, mais ce n'est pas mon premier choix; 4) oui, absolument. Un questionnaire sur les données sociologiques était aussi rempli par les participants ${ }^{30}$.

\subsection{Traitement des données évaluatives}

Les analyses statistiques ont été effectuées à l'aide du logiciel SPSS. Les réponses à la première question, sur la provenance perçue des locutrices, ont fait l'objet d'un dénombrement pour chaque locutrice isolément, puis pour chaque style, les pistes FOR regroupées d'un côté et les pistes INF de l'autre. Un test binomial a été effectué sur les pistes regroupées par style pour vérifier la significativité de la différence des réponses France versus Québec, en lien avec la probabilité qu'elles soient dues au hasard ou aux styles des pistes (Larson-Hall 2010: 210, 374, 390). Quant aux réponses sur la préférence de LC, elles ont été traitées avec des ANOVA à mesures répétées pour des comparaisons par paires entre chacune des pistes. Le choix de recourir à des ANOVA sur des données considérées continues est justifié,

28. Pourcentage moyen de la durée de l'assibilation sur la durée totale de la consonne pour [dy]: INF 64\%; FOR 54,9\%; [ti] : INF 64,6\%; FOR 55,6\%; [ty] : INF 69,2\%; FOR 68,4\%.

29. Deux items d'entrainement étaient d'abord présentés aux participants; les données n’ont été recueillies et analysées que sur les huit pistes sonores suivant les items d'entrainement.

30. Les consignes et les questionnaires ont été traduits du français vers l'espagnol et révisés par deux locuteurs hispanophones natifs d'Amérique latine ayant une bonne maitrise du français. 
d'abord par le fait que les observations sont faites sur des moyennes (Norman 2010) et, également, par le fait que la puissance des ANOVA demeure équivalente à celle de tests non paramétriques avec des échantillons de plus de trente (de Winter et Dodou 2010). L'examen de la normalité des distributions pour chaque piste a permis de constater qu'elles étaient dans la grande majorité des cas symétriques ${ }^{31}$.

\section{Résultats et discussion}

Les résultats des analyses, suivis d'une discussion, seront présentés en lien avec chaque question de recherche.

\subsection{Amalgame entre style et variété}

La première question de recherche était: «Les styles FOR et INF issus de chacune des deux variétés dialectales sont-ils associés par les participants au Québec ou à la France?»

\subsubsection{Résultats}

Les résultats des statistiques descriptives montrent des différences claires dans les réponses des participants pour certaines locutrices en fonction du style dans lequel elles ont été entendues. Le tableau 6 présente ces différences. On peut y lire que la locutrice Marie $(\mathrm{FF})$, en style INF, a été identifiée à la France par $53 \%$ des participants, alors qu'en style FOR, ce pourcentage augmente à $63 \%$. La locutrice française Amélie, quant à elle, a été perçue en style INF par $60 \%$ des participants comme une Québécoise, alors qu'en style FOR, elle est perçue comme une Française dans 70 \% des réponses. La locutrice québécoise Mireille, en style INF, a été identifiée comme Québécoise par $93 \%$ des participants, alors qu'en style FOR, ce pourcentage baisse à $58 \%$ des réponses. Finalement, Catherine (FQ) a été perçue en style INF comme une Québécoise dans $77 \%$ des réponses; lorsqu'elle se trouve en style FOR, ce pourcentage chute à $35 \%$, contre $65 \%$ qui l'ont identifiée à la France.

Tableau 6: Provenance perçue selon le style

\begin{tabular}{|c|c|c|c|c|c|c|c|}
\hline $\begin{array}{l}\text { Pistes } \\
\text { INF }\end{array}$ & Perception & $\mathbf{N}$ & $\%$ & $\begin{array}{l}\text { Pistes } \\
\text { FOR }\end{array}$ & Perception & $\mathbf{N}$ & $\%$ \\
\hline \multirow{3}{*}{$\begin{array}{l}\text { Marie, } \\
\text { FFinf }\end{array}$} & Québec & 20 & $47 \%$ & \multirow[t]{3}{*}{ Marie, FFfor } & Québec & 16 & $37 \%$ \\
\hline & France & 23 & $53 \%$ & & France & 27 & $63 \%$ \\
\hline & total & 43 & $100 \%$ & & total & 43 & $100 \%$ \\
\hline \multirow{3}{*}{$\begin{array}{l}\text { Amélie, } \\
\text { FFinf }\end{array}$} & Québec & 26 & $60 \%$ & \multirow{3}{*}{$\begin{array}{l}\text { Amélie, } \\
\text { FFfor }\end{array}$} & Québec & 13 & $30 \%$ \\
\hline & France & 17 & $40 \%$ & & France & 30 & $70 \%$ \\
\hline & total & 43 & $100 \%$ & & total & 43 & $100 \%$ \\
\hline \multirow{3}{*}{$\begin{array}{l}\text { Mireille, } \\
\text { FQinf }\end{array}$} & Québec & 40 & $93 \%$ & \multirow{3}{*}{$\begin{array}{l}\text { Mireille, } \\
\text { FQfor }\end{array}$} & Québec & 25 & $58 \%$ \\
\hline & France & 3 & $7 \%$ & & France & 18 & $42 \%$ \\
\hline & total & 43 & $100 \%$ & & total & 43 & $100 \%$ \\
\hline
\end{tabular}

31. Par mesure de précaution, un test non paramétrique (rangs signés de Wilcoxon) a été effectué pour les comparaisons par paires afin de contrevérifier les résultats des ANOVA à mesures répétées. Les résultats de ce test se sont avérés analogues à ceux des ANOVA. 


\begin{tabular}{|c|c|c|c|c|c|c|c|}
\hline $\begin{array}{l}\text { Pistes } \\
\text { INF }\end{array}$ & Perception & $\mathbf{N}$ & $\%$ & $\begin{array}{l}\text { Pistes } \\
\text { FOR }\end{array}$ & Perception & $\mathbf{N}$ & $\%$ \\
\hline \multirow{3}{*}{$\begin{array}{l}\text { Catherine, } \\
\text { FQinf }\end{array}$} & Québec & 33 & $77 \%$ & \multirow{3}{*}{$\begin{array}{l}\text { Catherine, } \\
\text { FQfor }\end{array}$} & Québec & 15 & $35 \%$ \\
\hline & France & 10 & $23 \%$ & & France & 28 & $65 \%$ \\
\hline & total & 43 & $100 \%$ & & total & 43 & $100 \%$ \\
\hline
\end{tabular}

Le regroupement des pistes selon le style, à savoir toutes les pistes FOR (FF et FQ) d'un côté et INF de l'autre, a aussi montré que la formalité de la situation orientait la provenance perçue par les participants. La figure 1 dénombre les attributions France ou Québec en fonction des styles FOR et INF.

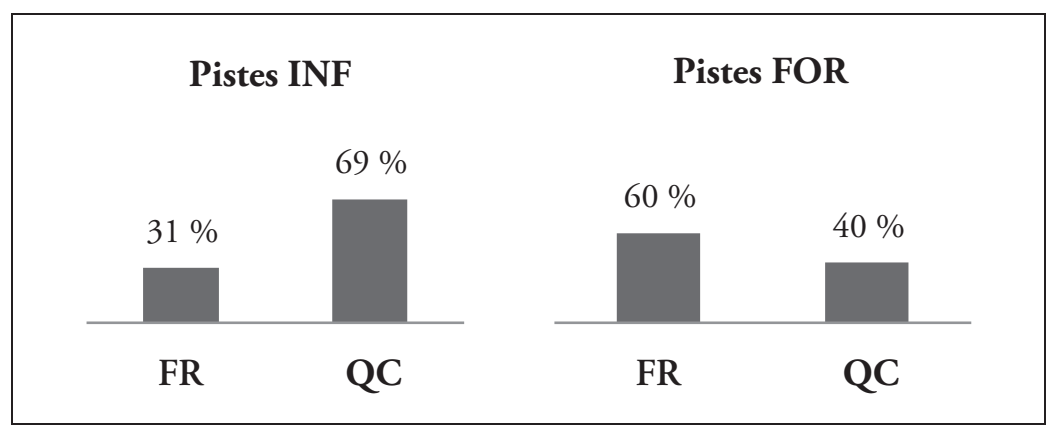

Figure 1: Pourcentage de réponses Fr/Qc en fonction des styles INF et FOR

Les pistes INF ont reçu $31 \%$ des réponses pour la provenance France et $69 \%$ pour la provenance Québec, tandis que les pistes FOR ont reçu $60 \%$ des réponses pour la provenance France, contre $40 \%$ pour la provenance Québec. Le test binomial de proportion a montré que les différences sont significatives à des seuils de $p=, 000$ pour les pistes INF et $p=, 012$ pour les pistes FOR.

Les attributions Québec ou France sont donc, au moins en partie, déterminées par les styles FOR ou INF des pistes. Les perceptions des participants, en ce qui a trait à la provenance des locutrices, se modifient en fonction de la variation stylistique. Cela apparait clairement à la figure 1 et au tableau 6 , entre les pistes FOR et INF des locutrices Amélie (FF), Mireille (FQ) et Catherine (FQ). Certains liens sont sans doute présents entre les variables linguistiques à l'étude et les variables attitudinales (jugements des participants). Comme indiqué à la section 5.2.4, la piste d'Amélie (FF) en situation INF, qui contient plus de variantes INF que celle de Marie (FF), a été perçue comme québécoise à $60 \%$ contre $47 \%$ pour celle de Marie. Il faut également souligner que Catherine (FQ), qui est perçue en situation FOR plutôt comme une Française (65\%), a montré un comportement plus normatif que Mireille (FQ). Finalement, Mireille (FQ), qui est la locutrice du FQ qui a le moins contrôlé de variables stylistiques et dialectales en situation FOR, demeure perçue plutôt comme une Québécoise (58 \%).

\subsubsection{Discussion}

Ces résultats corroborent les constats de Saint-Laurent (2008) et Calinon (2009), selon lesquels le FQ est amalgamé, dans les perceptions subjectives des participants, à un registre de langue non standard, alors que le FF est vu comme une norme de référence. Cependant, force est de constater une nuance importante: pour $40 \%$ des jugements émis sur les pistes FOR, les participants ont indiqué comme provenance le Québec. C'est donc dire que, pour certains apprenants de FLS, un français FOR peut être 
perçu comme du FQ. En dépit de cette nuance, la tendance est définitivement maintenue et le FQ ne semble avoir ni unanimement ni globalement obtenu le statut de langue standard, dans l'idée que s'en font les participants de l'étude. Si Archambault et Corbeil (1982) notent un manque véritable de modèle linguistique québécois dans les programmes et le matériel didactique en FLS, Calinon (2009) et Bigot et Papen (2013) le rapportent aussi, plus de 30 ans plus tard. L'enseignement, orienté vers un français de référence dont la norme est à tendance exogène (Calinon 2009) a peut-être en effet pour conséquence de transmettre aux apprenants une perception selon laquelle la variété du FQ est associée à une langue familière et populaire, alors que la langue «correcte» est associée au FF. Au même titre que Calinon (2009), Saint-Laurent (2008: 43) fait remarquer la (con)fusion entre niveau de langue («à ne pas atteindre») et variété dialectale («le français québécois») dans les commentaires des participants. Il semble que les apprenants de FLS de la présente étude tendent aussi à percevoir le style INF plutôt comme du FQ (à $69 \%$ ). De plus, comme le montre Kircher (2012), la variété du FF est, selon les allophones de son étude, généralement mieux évaluée sur le plan du statut social, vue comme plus prestigieuse et garante de meilleures possibilités sur le plan socioéconomique. Dans la mesure où les variantes FOR sont associées à plus de prestige (Labov 1972), tout comme le FF l'est en classe de FLS et chez les participants des études antérieures (Saint-Laurent 2008; Calinon 2009; Kircher 2012), il y a une cohérence dans le fait que, dans la présente étude, les styles FOR aient été associés plutôt au FF et les styles INF au FQ.

\subsection{Préférence de langue cible}

La seconde question à l'étude visait à connaitre la préférence des participants en matière de LC à l'égard de quatre possibilités, soit le FQ FOR et INF et le FF FOR et INF.

\subsubsection{Résultats}

Avec l'écoute des stimuli, l'énoncé Cette personne parle le français que je veux apprendre était à compléter par un des quatre choix de réponses suivants: non, pas du tout; non, pas vraiment; oui, mais ce n'est pas mon premier choix; oui, absolument. Les participants ont évalué chacune des huit pistes en cochant un des choix proposés. Les valeurs des réponses sont chiffrées de 1 (pour non, pas du tout) à 4 (pour oui, absolument) et des moyennes ont été calculées pour chaque piste en fonction des réponses de tous les participants. Ces résultats moyens sont présentés dans la figure 2. Plus le résultat moyen est élevé, plus la piste représente la LC.

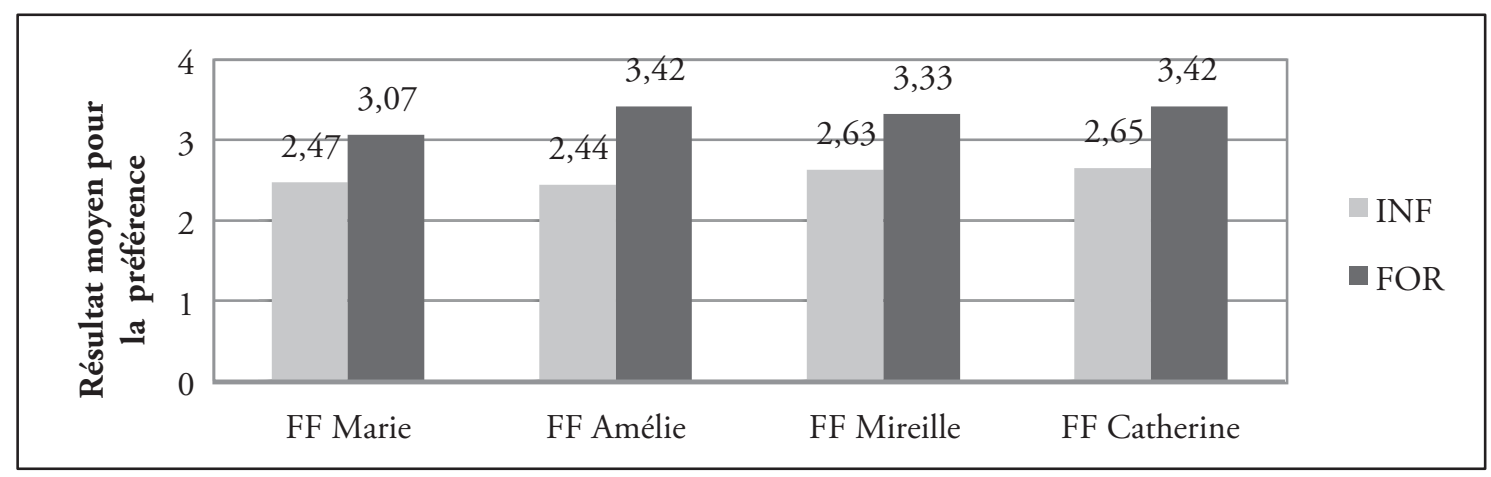

Figure 2: Moyennes pour la préférence de LC par locutrice et par style 
La figure 2 montre que les pistes ayant reçu les moyennes les plus élevées sont, à égalité $(3,42)$, les pistes FOR de Catherine (FQ) et d'Amélie (FF). La piste ayant reçu les résultats moyens les plus défavorables pour la préférence de LC $(2,44)$ est la piste INF d'Amélie (FF) qui, par ailleurs, a obtenu la moyenne la plus élevée en FOR. Les comparaisons par paires montrent que la différence entre la moyenne la plus haute et la plus basse $(3,42$ et 2,44$)$ est significative $(p=, 000)$. Aussi, les pistes FOR de Catherine (FQ) et d'Amélie (FF) ne sont pas évaluées de façon significativement différente des autres pistes FOR de FQ et de FF. Par contre, elles l'ont été de toutes les pistes INF (de $p=, 001$ à $p=, 000)$. D'un autre côté, la piste INF d'Amélie (FF), qui a le résultat moyen le plus bas, n'a pas été évaluée de façon significativement différente des autres pistes INF, mais l'a été de toutes les pistes FOR des deux variétés dialectales ( $\mathrm{de} p=, 003$ à $p=, 000$ ). Sur le plan de la préférence en matière de LC, toutes les locutrices ont été évaluées significativement différemment (entre $p=, 000$ et $p=, 006$ ), selon qu'elles sont en style FOR ou INF, peu importe leur variété dialectale. ${ }^{32}$ Autrement dit, dans un même style, qu'il soit FOR ou INF, les variétés dialectales n'ont pas entrainé de différences significatives pour la préférence de LC.

Tout comme pour la provenance des locutrices, c'est le style qui semble, en définitive, avoir eu un effet systématique sur les préférences des participants. Il est à souligner que, encore une fois, le comportement stylistique des locutrices peut, ici aussi, entretenir un rapport avec les préférences des participants. Catherine, la locutrice du FQ ayant eu un comportement plus normatif que Mireille (FQ) en situation FOR, obtient, au même titre qu'Amélie (FF) en situation FOR, la moyenne la plus élevée. Amélie (FF), en situation INF, se voit attribuer la moyenne la plus basse tout en étant la Française qui utilise le plus de variantes INF. Sans vouloir conclure à la variation stylistique comme seul facteur orientant les préférences des participants, les résultats indiquent très clairement une influence du style sur leurs choix.

\subsubsection{Discussion}

Par rapport aux statistiques rapportées par Maurais (2008), aux opinions recueillies par Saint-Laurent (2008) et Calinon (2009) et aux attitudes décrites par Kircher (2012), qui marquaient une tendance parfois nette à préférer le $\mathrm{FF}$ au $\mathrm{FQ}$, les résultats de la présente étude viennent proposer, non pas l'inverse, mais un portrait qui s'articule différemment. Si Kircher (2012) mentionne des attitudes plus favorables pour le FF en ce qui a trait au statut social et si les répondants de Maurais (2008) préfèreraient parler un FF à $63 \%$, le constat est ici nuancé: les résultats ex aequo des pistes FOR d'une Québécoise et d'une Française suggèrent que la LC préférée peut être à la fois québécoise et française (de France) mais surtout, qu'elle est avant tout une langue de registre FOR. Les résultats montrent que la LC la moins choisie, ou la plus souvent rejetée, est INF et issue des deux variétés dialectales.

Ceci étant dit, il m’apparait indispensable de mettre ce constat en relation avec les résultats de la perception de la provenance selon le style: non seulement les pistes FOR ont été associées au FF dans $60 \%$ des cas, mais les deux locutrices qui obtiennent les résultats les plus forts pour la préférence de LC, soit une Québécoise et une Française en situation FOR, sont aussi celles qui sont associées le plus souvent au FF (à $70 \%$ et $65 \%$ ). Par conséquent, il y a lieu d'avancer qu'une dynamique repérée chez Saint-Laurent (2008) et Calinon (2009) se répète dans la présente étude, sans pour autant être

32. Ceci confirme que les perceptions et préférences des participants n'ont pas été guidées par une préférence pour une voix en particulier, sinon, la même voix obtiendrait des évaluations semblables en situations FOR et INF, ce qui n'est pas le cas. 
catégorique: la LC est avant tout FOR, et cette langue FOR est perçue, la plupart du temps, comme provenant de France. Cette vision de la variété du FQ associée à un registre familier ou populaire, par rapport au FF, associé à la langue de référence, n'est pas sans rappeler la notion de diglossie selon laquelle deux (ou des) variétés de langue peuvent être investies de valeurs sociales différentes, notamment sur le plan du prestige (Ferguson 1959).

\section{Limites, pistes futures et conclusion}

Malgré le fait que des précautions aient été prises pour limiter la variation prosodique entre les pistes sonores (les situations et consignes suggéraient de "donner une information»), le rythme et l'intonation ont forcément subi des changements d'une piste à l'autre. Il serait intéressant de bâtir des stimuli dans lesquels une variation prosodique franche est présente afin d'en évaluer l'influence sur les perceptions.

D'un autre côté, la situation INF des enregistrements faits avec le test expressif ne donne peutêtre pas accès à la langue la moins surveillée des locutrices, même si les entrentiens ont été conduits dans une atmosphère de camaraderie laissant place à la spontanéité. À ce propos, il demeure important de considérer les résultats des préférences et des perceptions de la provenance en fonction du style. Ces résultats démontrent que le contraste de formalité a été perçu clairement et a orienté les choix des participants. Les styles FOR et INF sont possiblement en partie appréciables, dans la présente étude, dans une relation l'un par rapport à l'autre, plutôt que selon des formes catégoriques, figées ou prédéterminées.

Laur $(2008,2014)$ a montré que « [l] a voix d'une femme est [...] - d'une façon générale - perçue plus positivement que la voix d'un homme» (Laur 2008: 43). Pour avoir un portrait plus complet des perceptions globales du FQ et du FF, il serait pertinent de conduire une étude à la fois avec des voix d'hommes et de femmes. De plus, la même étude serait à reproduire avec d'autres groupes d'immigrants d'origine et de L1 différentes, afin de voir si et comment les différences entre ces groupes se profilent, le cas échéant.

Pour conclure, les résultats de la présente étude démontrent l'importance de la variation stylistique sur les perceptions et les préférences de LC, au-delà de celle de la variation dialectale. La technique du locuteur masqué, méthode indirecte d'investigation ici utilisée, a révélé des tendances très similaires à ce qu'ont pu observer auparavant, avec des méthodes directes, Maurais (2008), Saint-Laurent (2008) et Calinon (2009). Toutefois, elle aura peut-être apporté une vision plus détaillée des perceptions et des préférences de LC. Les analyses ont montré que les personnes immigrantes en francisation ayant participé à l'étude préferent une LC de style FOR à un style INF, autant pour le FQ que pour le FF. En parallèle, ces mêmes apprenants ont tendance à confondre partiellement le registre FOR avec la variété du FF (60 \%) et à amalgamer la variété du FQ à un registre familier (69\%). Ces constats reflètent une conception déjà véhiculée dans la société québécoise et dans les programmes de francisation en ce qui a trait à la valeur sociale associée au FQ (Calinon 2009; Bigot et Papen 2013) et suggèrent que la problématique demeure actuelle. Il est cependant important de rappeller qu'une partie des participants associe le style FOR au FQ (40 \%). Finalement, le contrôle qu'ont exercé les locutrices du FQ en situation FOR sur des variantes dialectales (relâchement des voyelles hautes et affrication) dont la stigmatisation ne s'avèrerait pourtant pas automatique et totale (Paradis et Dolbec 2008; Ostiguy et Toussigant 2008; Bigot et Papen 2013) invite à s'interroger davantage sur la représentation de la langue de référence chez les locuteurs natifs du FQ et sur la réflexion que pourrait poursuivre la société québécoise à propos de sa propre norme linguistique. 


\section{Références bibliographiques}

Archambault, A. et J.-C. Corbeil. 1982. L'enseignement du français langue seconde, aux adultes. Québec: Conseil supérieur de la langue française. Consulté le 20 avril 2016 de http://www.cslf.gouv.qc.ca/bibliothequevirtuelle/publication-html/?tx_iggcpplus_pi4\%5Bfile\%5D=publications/pubc122/c122ch1.html.

Bigot, D. 2011. «De la norme grammaticale du français parlé au Québec». Arborescences 1 : Identités linguistiques, langues identitaires: à la croisée du prescriptivisme et du patriotisme, sous la direction de A.-M. Brousseau. doi: http://dx.doi.org/10.7202/1001939ar.

Bigot, D. et R. A. Papen. 2013. «Sur la "norme” du français oral au Québec (et au Canada en général)». Langage et société 4 (146): 115-132. doi : 10.3917/ls.146.0115.

Blondeau, H. et M. Friesner. 2014. "Manifestations phonétiques de la dynamique des attributions ethnolinguistiques à Montréal». Canadian Journal of Linguistics/Revue canadienne de linguistique 59: 83-105. doi : http://dx.doi.org/10.1017/S0008413100000165.

Bordal, G. et C. Lyche. 2008. «La liaison en terre africaine». Communication présentée aux Journées PFC, décembre, Paris. Consulté le 3 juillet 2016 de http://www.projet-pfc.net/bulletins-et-colloques/colloqueset-journees-pfc/2008/journees-pfc-decembre-2008/51-bordal-lyche-la-liaison-en-terre-africaine.html.

Brasseur, A. 2009. Les marqueurs phonétiques de la perception de l'accent québécois. Mémoire de maitrise, Université du Québec à Montréal.

Brousseau, A.-M. 2011. "Identités linguistiques, langues identitaires: synthèse». Arborescences 1: Identités linguistiques, langues identitaires: à la croisée du prescriptivisme et du patriotisme, sous la direction de A.-M. Brousseau. doi : http://dx.doi.org/10.7202/1001938a.

Bouchard, C. 2002. La langue et le nombril: une histoire sociolinguistique du Québec. Montréal: Fides.

Boersma, P. et D. Weenink. 2016. Praat: Doing Phonetics by Computer [logiciel]. Version 6.0.19. Consulté le 3 juillet 2016 de http://www.praat.org/.

Bourdieu, P. 1982. Ce que parler veut dire: l'économie des échanges linguistiques. Paris: Fayard.

Calinon, A.-S. 2009. Facteurs linguistiques et sociolinguistiques de l'intégration en milieu multilingue: le cas des immigrants à Montréal. Thèse de doctorat, Université de Montréal, Canada, et Université de FrancheComté, France. Consulté le 20 mai 2016 de https ://papyrus. bib. umontreal.ca/xmlui/handle/1866/9122.

Cajolet-Laganière, H. et P. Martel. 1995. La qualité de la langue au Québec. Québec: Institut québécois de recherche sur la culture.

Cardinal, G. 2008. Le français langue seconde par thèmes. Niveau débutant. Cahier d'exercices, $2^{\mathrm{e}}$ édition. Montréal: Chenelière Éducation.

Conseil supérieur de la langue française (CSLF). 1990. L'aménagement de la langue: pour une description du français québécois. Québec: Gouvernement du Québec. Consulté le 20 mai 2016 de http://www.cslf. gouv.qc.ca/bibliotheque-virtuelle/publication-\%20html/?tx_iggcpplus_pi $4 \% 5 \mathrm{Bfile} \% 5 \mathrm{D}=$ publications/ avis116/a116.htm.

Conseil supérieur de la langue française (CSLF). 2007. Les accommodements raisonnables en matière linguistique: mémoire présenté à la Commission de consultation sur les pratiques d'accommodement reliées aux différences culturelles. Consulté le 20 mai 2016 de http://www.cslf.gouv.qc.ca/publications/pubf227/f227.pdf.

Côté, M.-H. 2004. "Consonant cluster simplification in Québec French". Probus 16: 151-201. doi: http:// dx.doi.org/10.1515/prbs.2004.16.2.151.

Côté, M.-H. 2012. "Laurentian French (Québec) : Extra vowels, missing schwas and surprising liaison consonants". Dans Phonological Variation in French: Illustrations from Three Continents, sous la direction de R. Gess, C. Lyche et T. Meisenburg. Amsterdam: John Benjamins: 235-274.

Côté, M.-H. 2014. "Liaison et assibilation en français laurentien». Dans La liaison: approches contemporaines, sous la direction de C. Soum-Favaro, A. Coquillon et J.-P. Chevrot. Berne: Peter Lang: 9-32.

Cox, T. B. 1998. "Vers une norme pour un cours de phonétique française au Canada». Canadian Modern Language Review 54: 172-197. doi : http://dx.doi.org/10.3138/cmlr.54.2.172. 
d'Anglejan, A. et R. Tucker. 1973. "Sociolinguistic correlates of speech style in Quebec". Dans Language Attitudes: Current Trends and Prospects, sous la direction de R. W. Shuy et R. W. Fasold. Washington (DC) : Georgetown University Press: 1-27.

Detey, S., J. Durand, B. Laks et C. Lyche, dir. 2010. Les variétés du français parlé dans l'espace francophone. Ressources pour l'enseignement. Paris: Ophrys.

de Winter, J. C. et D. Dodou. 2010. "Five-point Likert items: $t$ test versus Mann-Whitney-Wilcoxon”. Practical Assessment, Research and Evaluation 15 (11): 1-12.

Dumas, D. 1987. Nos façons de parler. Sillery: Presses de l'Université du Québec.

Durand, J. et C. Lyche. 2008. "French liaison in the light of corpus data". Journal of French Language Studies 18: 33-66. doi: http://dx.doi.org/10.1017/S0959269507003158.

Feagin, C. 2002. "Entering the community: Fieldwork". Dans The Handbook of Language Variation and Change, sous la direction de J. K. Chambers, P. Trudgill et N. Schilling-Estes. Malden (MA): Blackwell : 20-39.

Ferguson, C. A. 1959. "Diglossia”. Word 2: 325-340. doi: http://dx.doi.org/10.1080/00437956.1959.11659702.

Friesner, M. 2010. «Une prononciation "tsipéquement” québécoise? La diffusion de deux aspects stéréotypés du français canadien». Canadian Journal of Linguistics/Revue canadienne de linguistique 55: 27-53. doi: http:// dx.doi.org/10.1353/cjl.0.0070.

Gadet, F. 2007. La variation sociale en français, $2^{\mathrm{e}}$ édition. Paris: Orphys.

Gardner, R. C. 1985. Social Psychology and Second Language Learning: The role of Attitudes and Motivation. London: Edward Arnold.

Gardner, R. C., P. Tremblay et A. M. Masgoret. 1997. "Toward a full model of second language learning: An empirical investigation". The Modern Language Journal 81: 344-362. doi: http://dx.doi. org/10.1111/j.1540-4781.1997.tb05495.x.

Genessee F. et N. E. Holobow. 1989. "Change and stability in intergroup perception”. Journal of Language and Social Psychology 8 : 17-38. doi : http://dx.doi.org/10.1177/0261927X8900800102.

Giles, H. et P. F. Powesland. 1975. Speech Style and Social Evaluation. Londres: Academic Press.

Girard-Lamoureux, C. 2004. La langue d'usage public des allophones scolarisés au Québec. Québec: Conseil supérieur de la langue française. Consulté le 30 avril 2016 de http://bibvir1.uqac.ca/archivage/18347605.pdf.

Hansen, A. B. 2012. "A study of young Parisian speech: Some trends in pronunciation". Dans Phonological Variation in French: Illustration from Three Continents, sous la direction de R. Gess, C. Lyche et T. Meisenburg. Amsterdam: John Benjamins: 151-171.

Hansen, A. B. et C. Juillard. 2011. «La phonologie parisienneà trente ans d'intervalle : les voyelles à double timbre». Journal of French Language Studies 21 : 313-359. doi : http://dx.doi.org/10.1017/S0959269510000347.

Kircher, R. 2012. "How pluricentric is the French language? An investigation of attitudes towards Quebec French compared to European French”. Journal of French Language Studies 22: 345-370. doi: http:// dx.doi.org/10.1017/S0959269512000014.

Labov, W. 1972. Sociolinguistic Patterns. Philadelphie: University of Pennsylvania Press.

Labov, W. 2006. "A sociolinguistic perspective on sociophonetic research". Journal of Phonetics 34: 500-515. doi: http://dx.doi.org/10.1016/j.wocn.2006.05.002.

Lafontaine, D. 1986. Le parti pris des mots: normes et attitudes linguistiques. Bruxelles: Pierre Mardaga.

Lambert, W. E., R. C. Hodgson, R. C. Gardner et S. Fillembaum. 1960. "Evaluational reaction to spoken language". Journal of Abnormal and Social Psychology 60 (1): 44-51. doi: http://dx.doi.org/10.1037/ h0044430.

Lambert, W. E. 1967. "A social psychology of bilingualism”. The Journal of Social Issues 23: 91-109. doi : http:// dx.doi.org/10.1111/j.1540-4560.1967.tb00578.x.

Lappin, K. 1982. «Évaluation de la prononciation du français montréalais: étude sociolinguistique». Revue québécoise de linguistique 11 : 93-112. doi: http://dx.doi.org/10.7202/602489ar.

Larson-Hall, J. 2010. A Guide to Doing Statistics in Second Language Research Using SPSS. New York: Routledge. 
Lasagabaster, D. 2006. «Les attitudes linguistiques: un état des lieux». Éla. Études de linguistique appliquée 4: 393-406. Consulté le 20 avril 2016 de https:/www.cairn.info/revue-ela-2006-4-page-393.htm.

Laur, E. 2008. Contribution à l'étude des perceptions linguistiques. La méthodologie des faux-couples revisitée. Québec: Gouvernement du Québec.

Laur, E. 2014. "Evaluational reactions to spoken French and English in Montreal: Does mother tongue really matter?". Canadian Journal of Linguistics/Revue canadienne de linguistique 59: 3-23. doi: http://dx.doi. org/10.1017/S000841310000013X.

Lepicq, D. et R. Bourhis. 1995. "Aménagement linguistique et norme langagière au Québec». Linx 33: 109128. doi : http://dx.doi.org/10.3406/linx.1995.1396.

Lyche, C., T. Meisenburg et R. Gess. 2012. "Phonological variation in French: Unity and diversity across continents". Dans Phonological Variation in French: Illustrations from Three Continents, sous la direction de R. Gess, C. Lyche et T. Meisenburg. Amsterdam: John Benjamins: 369-388.

Maurais, J. 2008. Les Québécois et la norme: l'évaluation par les Québécois de leurs usages linguistiques. Québec: Office québécois de la langue française, Gouvernement du Québec.

Mazzoni, D. et R. Dannenbergen. 1999. Audacity [logiciel]. Consulté le 30 avril 2016 de http://www. audacityteam.org/

Ministère de l'Immigration, de la Diversité et de l'Inclusion (MIDI). 2014. Portraits statistiques. L'immigration permanente au Québec selon les catégories d'immigration et quelques composantes 2009-2013. Québec: Gouvernement du Québec.

Ministère de l'Immigration, de la Diversité et de l'Inclusion. 2016. Connaissances en français et en anglais pour les candidats du Programme régulier des travailleurs qualifiés. Consulté le 20 avril 2016 de http:// www.immigration-quebec.gouv.qc.ca/fr/immigrer-installer/travailleurs-permanents/conditions-requises/ connaissances-linguistiques.html.

Moreau, M.-L. 1990. "Des pilules et des langues: le volet subjectif d'une situation de multilinguisme au Sénégal». Dans Des langues et des villes, sous la direction de E. Gouaini et N. Thiam. Paris: Didier Érudition: 407-420.

Morin, Y.-C. 2009. "À propos de la fermeture des voyelles moyennes devant [r] dans le français du Québec». Canadian Journal of Linguistics/Revue canadienne de linguistique 54: 461-510. doi: http://dx.doi. org/10.1017/S0008413100004618.

Morin, Y.-C., M.-C. Langlois et M.-E. Varin. 1990. "Tensing of word-final [short open back vowel o] to [o] in French: The phonologization of a morphophonological rule”. Romance Philology 43: 507-528.

Mougeon, F. 1995. Quel français parler? Initiation au français parlé au Canada et en France. Toronto: Éditions du Gref, Centre Universitaire Glendon, Université York.

Mougeon, R. et É. Beniak. 1994. Les origines du français québécois. Québec: Presses de l'Université Laval.

Moyer, A. 2013. Foreign Accent: The Phenomenon of Non-Native Speech. Cambridge: Cambridge University Press.

Norman, G. 2010. "Likert scales, levels of measurement and the "laws" of statistics". Advances in Health Sciences Education 15: 625-632. doi: http://dx.doi.org/10.1007/s10459-010-9222-y.

Ostiguy, L. et C. Tousignant. 2008. Les prononciations du français québécois: normes et usages, $2^{\mathrm{e}}$ édition. Montréal: Guérin.

Paradis, C. et J. Dolbec. 2008. Les principales caractéristiques phonétiques du français parlé au Québec. Université du Québec à Chicoutimi. Consulté le 20 avril 2016 de http://phono.uqac.ca/index.php?article=accueil

Paternostro, R. 2014. "L'éveil à la variation phonétique en didactique du français langue étrangère: enjeux et outils». Lidil 50 : 105-124.

Pupier, P. et L. Drapeau. 1973. «La réduction des groupes de consonnes finales en français de Montréal». Cahier de linguistique 3: 127-145. doi: http://dx.doi.org/10.7202/800025ar.

Reinke, K. 2005. La langue à la télévision québécoise: aspects sociophonétiques. Québec: Gouvernement du Québec. 
Saint-Laurent, N. 2008. Le français et les jeunes. Québec: Conseil supérieur de la langue française. Consulté le 20 avril 2016 de http://www.cslf.gouv.qc.ca/publications/pubf223/f223.pdf.

Santerre, L. 1974. "Deux E et deux A phonologiques en français québécois: étude phonologique, articulatoire et acoustique des oppositions de timbre et de durée». Cahier de linguistique 4: 117-145. doi: http:// dx.doi.org/10.7202/800031ar.

Statistique Canada. 2011. Enquête nationale auprès des ménages de 2011. Compilation de Montréal en statistiques. Montréal: Annuaire statistique de l'agglomération de Montréal.

Thomas, A. 1989. «La prononciation du /a/ final en franco-ontarien». Dans Le français canadien parlé hors Québec: aperçu sociolinguistique, sous la direction de R. Mougeon et É. Beniak. Québec: Presses de l'Université Laval: 53-67.

Thomas, E. R. 2002. "Sociophonetic applications of perception experiments". American Speech 77: 115-147. doi: 10.1215/00031283-77-2-115

Tremblay, P. et R. C. Gardner. 1995. "Expanding the motivation construct in language learning”. The Modern Language Journal 79: 505-518. doi : http://dx.doi.org/10.1111/j.1540-4781.1995.tb05451.x.

Valdman, A. 2000. «Comment gérer la variation dans l'enseignement du français langue étrangère aux ÉtatsUnis». The French Review 73: 648-666.

Walker, D. C. 2005. "Le français dans l'Ouest canadien». Dans Le français en Amérique du Nord: état présent, sous la direction de A. Valdman, J. Auger et D. Piston-Hatlen. Québec: Presses de l'Université Laval: 187-205.

Walter, H. 1982. Enquête phonologique et variétés régionales du français. Paris: Presses Universitaires de France. 